\title{
Electronic quantum Monte Carlo calculations of atomic forces, vibrations, and anharmonicities
}

\author{
Myung Won Lee \\ The Makineni Theoretical Laboratories, Department of Chemistry, University of Pennsylvania, Philadelphia, \\ Pennsylvania 19104-6323 \\ Massimo Mella \\ School of Chemistry, Cardiff University, P.O. Box 912, Cardiff CF10 3TB, United Kingdom \\ Andrew M. Rappe ${ }^{\text {a) }}$ \\ The Makineni Theoretical Laboratories, Department of Chemistry, University of Pennsylvania, Philadelphia, \\ Pennsylvania 19104-6323
}

(Received 22 November 2004; accepted 7 April 2005; published online 24 June 2005)

\begin{abstract}
Atomic forces are calculated for first-row monohydrides and carbon monoxide within electronic quantum Monte Carlo (QMC). Accurate and efficient forces are achieved by using an improved method for moving variational parameters in variational QMC. Newton's method with singular value decomposition (SVD) is combined with steepest-descent (SD) updates along directions rejected by the SVD, after initial SD steps. Dissociation energies in variational and diffusion QMC agree well with the experiment. The atomic forces agree quantitatively with potential-energy surfaces, demonstrating the accuracy of this force procedure. The harmonic vibrational frequencies and anharmonicity constants, derived from the QMC energies and atomic forces, also agree well with the experimental values. (C) 2005 American Institute of Physics. [DOI: 10.1063/1.1924690]
\end{abstract}

\section{INTRODUCTION}

Quantum Monte Carlo (QMC) is an effective method for solving the time-independent Schrödinger equation and has become quite successful in computing ground-state total energies. The QMC method gives energies of atoms, molecules, and solids that are comparably accurate or more accurate than traditional techniques such as density-functional theory (DFT), multiconfiguration self-consistent field (MCSCF), or coupled cluster methods. Although the situation for the calculation of properties other than energies has been less favorable, the accurate QMC calculation of atomic forces has been enabled through the recent developments made in this area by Assaraf and Caffarel, ${ }^{1,2}$ Filippi and Umrigar, ${ }^{3}$ Casalegno et al., ${ }^{4}$ Chiesa et al., ${ }^{5}$ and others.

In this paper, we extend our atomic force methodology to all the first-row monohydrides and carbon monoxide. In order to acquire energies and forces efficiently for these systems, we also describe an improved algorithm for optimizing variational Monte Carlo (VMC) wave functions. As in our previous paper, ${ }^{6}$ the first and second derivatives of the variational energy are analytically computed and used to perform Newton's method parameter updates with singular value decomposition (SVD). We now propose augmenting this approach by using the steepest-descent (SD) method in the subspace neglected by Newton's method with SVD. In the initial stage of parameter update, Newton's method might perform poorly since the second derivatives include larger noise when the parameters are far from the optimum. So we take two SD steps before starting Newton's method. The improved algo-

${ }^{a)}$ Electronic mail: rappe@sas.upenn.edu rithm was applied to the calculation of the ground-state energies and forces of the first-row monohydrides and carbon monoxide. In general, the direct application of the variational principle yields significantly lower energy than variance minimization methods, so minimizing the energy is advantageous. The wave functions optimized in VMC were used as a guiding function to compute more accurate energies and forces in diffusion Monte Carlo (DMC).

In this paper, total energies, dissociation energies, forces, harmonic vibrational frequencies, and anharmonicity constants are reported for all first-row monohydrides from $\mathrm{LiH}$ to $\mathrm{HF}$, as well as for $\mathrm{CO}$. In all cases, the computed results agree well with the experiment. The dissociation energies in VMC are significantly improved with respect to a previous VMC study of the hydrides.

\section{THEORETICAL BACKGROUND AND COMPUTATIONAL DETAILS}

The variational parameters used in VMC will be denoted as $c_{1}, c_{2}, \ldots, c_{n}$, here. The VMC energy expectation value $E_{T}$ is a function of these variational parameters, and the parameter set that minimizes $E_{T}$ is sought.

The SD method is useful in the initial stages of parameter optimization in VMC, due to the large error bars of Hessian matrix components. One arbitrary constant is necessary to implement the SD method. We used the following two-step scheme to find a good SD constant.

Let $\mathbf{Q}_{0}$ and $\mathbf{Q}$ be the vectors composed of variational parameters before and after update, respectively,

$$
\mathbf{Q}_{0}=\left(c_{1,0} c_{2,0} \cdots c_{n, 0}\right)^{T},
$$




$$
\mathbf{Q}=\left(c_{1} c_{2} \cdots c_{n}\right)^{T},
$$

and let $\mathbf{g}$ be the gradient vector of energy with respect to the variational parameters,

$$
\mathbf{g}=\left(g_{1} g_{2} \cdots g_{n}\right)^{T}=\left(\frac{\partial E}{\partial c_{1}} \frac{\partial E}{\partial c_{2}} \cdots \frac{\partial E}{\partial c_{n}}\right)^{T} .
$$

In the first update, a value $a^{(0)}$ is chosen as a SD constant, which is small enough not to exhaust the downhill direction,

$$
\mathbf{Q}=\mathbf{Q}_{0}-a^{(0)} \mathbf{g}\left(\mathbf{Q}_{0}\right) \text {. }
$$

After the first update, the VMC simulation is performed again to get the gradient at the new parameter set $\mathbf{Q}$. If we consider only the $i$ th component, the best value for the new SD constant $a_{i}^{(1)}$ will make the gradient component $g_{i}$ zero in the next simulation and will be given by

$$
a_{i}^{(1)}=\frac{a^{(0)}}{1-g_{i}(\mathbf{Q}) / g_{i}\left(\mathbf{Q}_{0}\right)} .
$$

Although $a_{i}^{(1)}$ values are different from component to component, it is usually the case that they are quite similar. So the averaged value was used for the next update,

$$
a^{(1)}=\frac{1}{n} \sum_{i=1}^{n} a_{i}^{(1)}
$$

These two steps of parameter updates using SD reduce the energy enough to greatly reduce the error bars, enabling Newton's method.

If we let $\mathbf{H}$ represent the Hessian matrix, the parameters can be updated according to Newton's method,

$$
\mathbf{Q}=\mathbf{Q}_{0}-\mathbf{H}^{-1}\left(\mathbf{Q}_{0}\right) \mathbf{g}\left(\mathbf{Q}_{0}\right) \text {. }
$$

Since $\mathbf{H}\left(\mathbf{Q}_{0}\right)$ and $\mathbf{g}\left(\mathbf{Q}_{0}\right)$ are calculated in the VMC simulation, we must invert $\mathbf{H}\left(\mathbf{Q}_{0}\right)$ for Newton's method update of the parameters.

It is well known that any matrix, e.g., $\mathbf{H}$, can be expressed as

$$
\mathbf{H}=\mathbf{U}\left[\operatorname{diag}\left(w_{j}\right)\right] \mathbf{V}^{T},
$$

where $w_{j} \geqslant 0$ and $\mathbf{U}, \mathbf{V}$ are orthogonal. ${ }^{7}$ For a square matrix, the inverse matrix can be obtained by

$$
\mathbf{H}^{-1}=\mathbf{V}\left[\operatorname{diag}\left(1 / w_{j}\right)\right] \mathbf{U}^{T} .
$$

Very small values of $w_{j}$ lead to erroneous moves along the directions corresponding to these components due to large $1 / w_{j}$ terms. For that reason, if $w_{j}$ is less than a certain threshold value, $1 / w_{j}$ is set to 0 in the actual calculation (SVD).

The SVD method has been tested for the inversion of the Hessian matrix, and it consistently gives robust results in many cases. However, the SVD method, by zeroing out small $w_{j}$ values, is equivalent to abandoning the corresponding search directions, the use of which may give better results. So we propose a modified algorithm in which the SD method is added for components discarded in the SVD method.

If we let $\mathbf{U}$ and $\mathbf{V}$ be equal to the square matrix whose column vectors are the normalized eigenvectors of $\mathbf{H},\left\{w_{j}\right\}$ will be the eigenvalues of $\mathbf{H}$. For values of $w_{j}$ that are smaller than the threshold, $1 / w_{j}$ can be replaced by a constant $a$ instead of zero, which is equivalent to the SD method along the corresponding directions. This method makes it possible to use the information for all directions, some of which are discarded in the SVD method, and it can be beneficial in cases where some eigenvalues of the Hessian matrix become close to zero, due to the noise inherent in QMC. In the standard SVD algorithm, $w_{j}$ is always non-negative, which corresponds to the absolute value of eigenvalue of $\mathbf{H}$. If any eigenvalue is negative and its absolute value is larger than the threshold, there is a problem that the direction corresponding to this is not discarded, even though this does not happen so frequently. This small problem of negative eigenvalues can be handled by using the modified method with the same positive threshold and zero steepest-descent constant, and we used this modified method in the actual implementation.

To construct the trial wave functions used in VMC, the following method was used. First, a contracted Gaussiantype function (CGTF) was fitted to each Slater-type orbital (STO). Ten primitive Gaussians were used for $1 s-$, eight for $2 s-$ or $2 p-$, and six for $3 s-, 3 p-$, or $3 d$-type STOs. The orbital exponents of STOs in the works of Cade and Huo ${ }^{8,9}$ were adopted (excluding the $f$-type orbitals). For the first-row monohydrides, each first-row atom has 29 STOs centered on it $\left(1 s, 1 s^{\prime}, 2 s, 2 s^{\prime}, 3 s\right.$, three $2 p$ 's, three $2 p^{\prime}$ 's, three $2 p^{\prime \prime \prime}$ 's, three $3 p$ 's, six $3 d$ 's, and six $3 d^{\prime}$ 's for $\mathrm{Li}$, and $1 s, 1 s^{\prime}, 2 s, 2 s^{\prime}, 3 s$, three $2 p$ 's, three $2 p^{\prime}$ 's, three $2 p^{\prime \prime}$ 's, three $2 p^{\prime \prime \prime \prime}$ 's, six $3 d$ 's, and six $3 d^{\prime}$ 's for other first-row atoms), and each hydrogen atom has 6 STOs centered on it $\left(1 s, 1 s^{\prime}, 2 s\right.$, and three $2 p$ 's) as a basis set. For carbon monoxide, each atom has 19 STOs centered on it $\left(1 s, 1 s^{\prime}, 2 s, 3 s\right.$, three $2 p$ 's, three $2 p^{\prime}$ 's, three $2 p^{\prime \prime}$ 's, and six $3 d$ 's) as a basis set.

Each molecular orbital (MO) was expressed as a linear combination of STOs, the coefficients of which were obtained using the Hartree-Fock method in GAUSSIAN 98 (G98). ${ }^{10}$ For the open shell molecules, restricted open shell Hartree-Fock (ROHF) wave functions were used. The MOs from G98 were used to construct the Slater determinants for $\alpha$ and $\beta$ electrons. While multideterminant trial wave functions give improved results for some systems, it was reported that the use of single determinant trial wave functions gave good results in the calculations of the first-row hydrides. ${ }^{11,12}$ Since the use of multideterminant trial wave functions is much more time consuming, we used a single determinant in the calculations presented. The product of a spin-up and a spin-down determinant was multiplied by a positive correlation factor to form a trial wave function, 13,14

$$
\Psi_{T}=D^{\uparrow} D^{\downarrow} \exp \left(\sum_{a, i<j} U_{a i j}\right),
$$

where

$$
U_{a i j}=\sum_{k}^{N_{a}} c_{k a}\left(\vec{r}_{a i}^{l_{k a}} \vec{r}_{a j}^{m_{k a}}+\vec{r}_{a j}^{l_{k a}} \vec{r}_{a i}^{m_{k a}}\right) \vec{r}_{i j}^{n_{k a}} .
$$

In this equation, $a$ and $i, j$ refer to the nuclei and the electrons, respectively, and $\bar{r} \equiv b r /(1+b r) . c_{k a}$ 's are variational Jastrow parameters. We used $b=1 a_{0}^{-1}$ and included 30 terms 


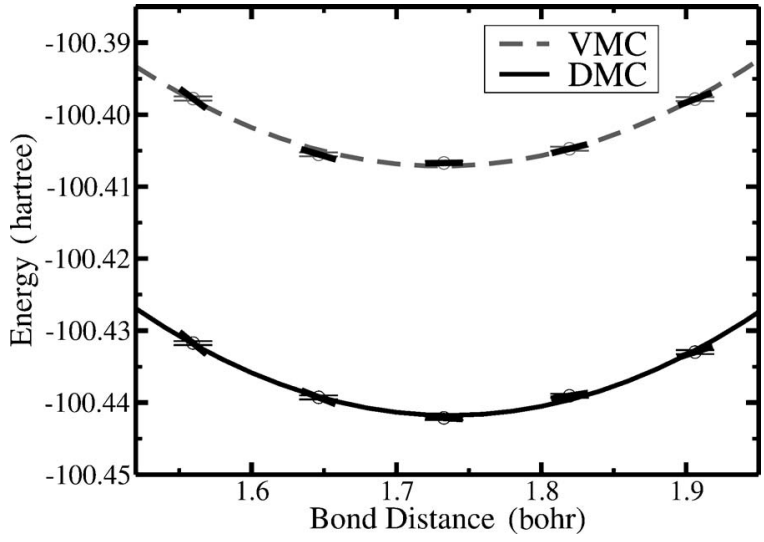

FIG. 1. Energy and force calculations of HF with VMC and DMC. Two thin horizontal lines at each data point show the energy error bar. The slope of the thick lines show the force at each data point.

for diatomic molecules, namely, 4 electron-electron, 6 electron-nucleus, and 20 electron-electron-nucleus terms. For single-atom calculations, we used 17 parameters composed of 4 electron-electron, 3 electron-nucleus, and 10 electronelectron-nucleus terms, to be consistent with the calculation of diatomic molecules.

Five different bond distances around the experimental bond length were used for calculation, namely, 90\%, 95\%, $100 \%, 105 \%$, and $110 \%$ of the experimental bond length $r_{\text {exp }}$. Two thousand walkers were used for all the calculations in this paper. In updating Jastrow parameters, average over 100 blocks was made, where each block was the average over 100 steps. To accelerate the sampling, a Fokker-Planck-type equation was used. ${ }^{15}$

After a short initial simulation without Jastrow factor, the Hartree-Fock wave function was multiplied by the Jastrow factor with all parameters set to zero. The gradient and Hessian of energy with respect to the Jastrow parameters were computed in the VMC simulation after this step. Using the gradient and Hessian information, a new Jastrow parameter set is calculated, and a new VMC simulation is performed with this updated parameter set. This process was iterated until the energy converged. Fully optimized parameters were obtained by 10-15 iterations. One iteration took about $30 \mathrm{~min}$ for $\mathrm{LiH}$ and about $90 \mathrm{~min}$ for $\mathrm{HF}$ when a single 2.8-GHz Intel@ Xeon ${ }^{\mathrm{TM}}$ processor was used.

After optimizing the trial wave function using VMC, a fixed-node DMC calculation was performed using importance sampling, as proposed by Reynolds et al. ${ }^{16}$ The DMC time step was 0.005 a.u. for the first-row hydrides and 0.0005-0.001 a.u. for carbon monoxide. A similar DMC method was used by Lüchow and Anderson ${ }^{11,17}$ in their calculation of first-row hydrides.

Force calculations were performed in both VMC and DMC. We followed the method described previously. ${ }^{4}$ If the wave function is exact, the Hellmann-Feynman theorem (HFT) would give the exact force. Since the trial wave function $\Psi_{T}$ is not exact, the terms that cancel in the case of exact wave functions should be considered, in addition to the HFT expression. Retaining terms involving wave-function derivatives gives the total atomic force on atom $a$ in direction $q$,
TABLE I. $E_{0}, r_{e}, \omega_{e}$, and $\omega_{e} x_{e}$ for $\mathrm{LiH}-\mathrm{HF}$ and $\mathrm{CO}$ obtained from VMC and DMC calculations and experimental data.

\begin{tabular}{|c|c|c|c|c|c|}
\hline & & $E_{0}($ hartree $)$ & $r_{e}($ bohr $)$ & $\omega_{e}\left(\mathrm{~cm}^{-1}\right)$ & $\omega_{e} x_{e}\left(\mathrm{~cm}^{-1}\right)$ \\
\hline \multirow[t]{3}{*}{$\mathrm{LiH}$} & VMC & -8.063 & $3.038(1)$ & $1402(4)$ & $25.7(1)$ \\
\hline & DMC & -8.070 & $3.020(1)$ & $1417(4)$ & $24.8(1)$ \\
\hline & Exp & -8.070 & 3.015 & 1406 & 23.2 \\
\hline \multirow[t]{3}{*}{$\mathrm{BeH}$} & VMC & -15.235 & $2.519(1)$ & 2141(4) & $56.6(2)$ \\
\hline & DMC & -15.246 & $2.515(1)$ & 2134(4) & $58.5(2)$ \\
\hline & Exp & -15.248 & 2.537 & 2061 & 36.3 \\
\hline \multirow[t]{3}{*}{$\mathrm{BH}$} & VMC & -25.254 & $2.370(1)$ & $2332(5)$ & $47.0(2)$ \\
\hline & DMC & -25.275 & $2.386(1)$ & $2369(5)$ & $47.3(2)$ \\
\hline & Exp & -25.289 & 2.329 & 2367 & 49.4 \\
\hline \multirow[t]{3}{*}{$\mathrm{CH}$} & VMC & -38.438 & $2.097(1)$ & 2961(6) & $77.2(3)$ \\
\hline & DMC & -38.463 & $2.112(1)$ & $2898(6)$ & $71.8(3)$ \\
\hline & Exp & -38.490 & 2.116 & 2858 & 63.0 \\
\hline \multirow[t]{3}{*}{$\mathrm{NH}$} & VMC & -55.178 & $1.941(1)$ & $3415(7)$ & $104.3(4)$ \\
\hline & DMC & -55.206 & $1.962(1)$ & $3253(7)$ & $92.0(4)$ \\
\hline & Exp & -55.247 & 1.958 & 3282 & 78.4 \\
\hline \multirow[t]{3}{*}{$\mathrm{OH}$} & VMC & -75.687 & $1.820(1)$ & $3854(7)$ & $101.2(4)$ \\
\hline & DMC & -75.720 & $1.843(1)$ & $3690(7)$ & $91.4(4)$ \\
\hline & Exp & -75.778 & 1.832 & 3738 & 84.9 \\
\hline \multirow[t]{3}{*}{$\mathrm{HF}$} & VMC & -100.407 & $1.729(1)$ & $4206(9)$ & $89.9(4)$ \\
\hline & DMC & -100.442 & $1.755(1)$ & $4040(9)$ & $82.4(4)$ \\
\hline & $\operatorname{Exp}$ & -100.531 & 1.733 & 4138 & 89.9 \\
\hline \multirow[t]{3}{*}{$\mathrm{CO}$} & VMC & -113.176 & $2.095(1)$ & $2539(16)$ & $21.1(3)$ \\
\hline & DMC & -113.286 & $2.116(2)$ & $2251(26)$ & $14.2(3)$ \\
\hline & Exp & -113.377 & 2.132 & 2170 & 13.3 \\
\hline
\end{tabular}

$$
F_{q a}=F_{q a}^{\mathrm{HFT}}+F_{q a}^{\text {Pulay }}+F_{q a}^{c},
$$

where

$$
\begin{aligned}
F_{q a}^{\mathrm{HFT}}= & -\frac{\left\langle\Psi_{T}\left|\frac{\partial \hat{H}}{\partial R_{q a}}\right| \Psi_{T}\right\rangle}{\left\langle\Psi_{T} \mid \Psi_{T}\right\rangle}, \\
F_{q a}^{\text {Pulay }}=-2 \frac{\left.\partial \Psi_{T}|\hat{H}| \Psi_{T}\right\rangle}{\partial R_{q a} \mid} \mid\left\langle\frac{\partial \Psi_{T} \mid}{\partial R_{q a}} \mid \Psi_{T}\right\rangle & \left\langle\Psi_{T} \mid \Psi_{T}\right\rangle
\end{aligned}
$$

and

$$
F_{q a}^{c}=-\sum_{k} \frac{\partial c_{k}}{\partial R_{q a}} \frac{\partial\langle E\rangle_{\mathrm{VMC}}}{\partial c_{k}} .
$$

These expressions apply for VMC, and similar equations are used for DMC simulations. ${ }^{4} F_{q a}^{\text {Pulay }}$ incorporates the explicit dependence of the wave function on the nuclear coordinates (Pulay's correction ${ }^{18}$ ) and can be easily calculated through VMC or DMC simulations. $F_{q a}^{c}$ depends implicitly on the nuclear coordinates through the variational parameters. However, since an energy-minimized wave function is used, i.e., $\partial\langle E\rangle_{\mathrm{VMC}} / \partial c_{k}=0$, this force term makes zero contribution. In 
TABLE II. Dissociation energies $D_{e}$ in $\mathrm{kcal} / \mathrm{mol}$ for $\mathrm{LiH}-\mathrm{HF}$ and $\mathrm{CO}$ obtained from our QMC calculation and from the literature.

\begin{tabular}{lccccc}
\hline \hline & VMC $^{\mathrm{a}}$ & DMC $^{\mathrm{a}}$ & VMC $^{\mathrm{b}}$ & DMC $^{\mathrm{b}}$ & Experiment $^{\mathrm{b}}$ \\
\hline $\mathrm{LiH}$ & 54.7 & 57.8 & 45.7 & 57.8 & 58.0 \\
$\mathrm{BeH}$ & 57.9 & 55.7 & 49.4 & 52.1 & 49.8 \\
$\mathrm{BH}$ & 82.7 & 84.7 & 63 & 84.8 & 84.1 \\
$\mathrm{CH}$ & 81.1 & 83.5 & 81 & 83.9 & 83.9 \\
$\mathrm{NH}$ & 80.2 & 82.3 & 77 & 81.4 & $80.5-84.7$ \\
$\mathrm{OH}$ & 105.1 & 106.4 & 86 & 106.4 & 106.6 \\
$\mathrm{HF}$ & 140.4 & 141.4 & 130 & 141.3 & 141.5 \\
$\mathrm{CO}$ & 218.1 & 254.9 & $\cdots$ & $\cdots$ & 258.7 \\
\hline \hline
\end{tabular}

$\overline{\bar{a} \text { Differences between QMC energies of molecules in Table I and QMC energies of atoms calculated with } 17}$ parameters.

${ }^{\mathrm{b}}$ From Ref. 11 for first-row hydrides.

the calculation of the Hellmann-Feynman theorem force $F_{q a}^{\mathrm{HFT}}$, the renormalized estimator proposed by Assaraf and Caffarel ${ }^{1}$ was used to reduce the variance of the force calculation. The expectation value of this estimator $F_{q a}^{\mathrm{AC}}$ is the same as $F_{q a}^{\mathrm{HFT}}$, but the variance of the former is much smaller. In our force calculation, $F_{q a}^{\mathrm{AC}}+F_{q a}^{\text {Pulay }}$ was computed by averaging over the walkers.

\section{RESULTS AND DISCUSSION}

The energies of first-row monohydrides and carbon monoxide at various bond distances were calculated. The plot of energy versus bond distance for hydrogen fluoride (HF) is shown in Fig. 1. In obtaining each point, 1000 blocks, each of which was composed of 100 steps, were used with the optimized Jastrow parameters. The plots for other molecules are similar to that for HF. The energies obtained from VMC are a few tenths of a Hartree lower than the Hartree-Fock energies obtained from G98, so the Hartree-Fock results are not shown in the figure. It can be seen from Table I that the DMC energy is significantly lower than the VMC energy and is close to the experimental value.

The bond dissociation energies $D_{e}$ were calculated by taking the differences between QMC energies of diatomic molecules in Table I and QMC energies of atoms. To be consistent in the number of the Jastrow parameters, we did the calculation of atoms with 17 parameters. The VMC en- ergies of atoms with 17 parameters fall between those with 9 parameters and those with 42 parameters reported in Ref. 6 . The dissociation energies are summarized in Table II, together with the results given in the work by Lüchow and Anderson. ${ }^{11}$ Our VMC dissociation energies are much closer to the experimental values than those given by Lüchow and Anderson, while our DMC results are quite similar to theirs. The improvement in our VMC results may be attributed to the effectiveness of the energy minimization method relative to the variance minimization method used for the VMC calculations in Ref. 11, while part of the improvement is also due to the larger number of the Jastrow parameters in our calculation.

The energies calculated by DMC are quite close to the experimental values for lighter first-row hydrides, while slightly higher energies than the experimental values are obtained for heavier molecules. This may be due to the approximations used in the DMC calculations: fixed-node approximation, neglect of the relativistic effect, and the error related with finite time step. To estimate the finite time step error, the DMC calculations at $r_{\exp }$ with several different time step values ranging from 0.0001 to 0.005 a.u. were carried out for the first-row hydrides. All calculated energies agreed within 2-3 mhartree.

In the VMC calculation of HF, the Jastrow parameter set at $r_{\exp }$ was optimized first, and after the optimization at this

TABLE III. The cosine similarity values between Jastrow parameter sets obtained from the VMC calculations of $\mathrm{HF}$ and $\mathrm{CH}$.

\begin{tabular}{lccccc}
\hline \hline $\cos \theta(\mathrm{HF})$ & $0.90 r_{\exp }$ & $0.95 r_{\exp }$ & $1.00 r_{\exp }$ & $1.05 r_{\exp }$ & $1.10 r_{\exp }$ \\
\hline $0.90 r_{\exp }$ & 1.000 & & & & \\
$0.95 r_{\exp }$ & 0.998 & 1.000 & & & \\
$1.00 r_{\exp }$ & 0.997 & 1.000 & 1.000 & & \\
$1.05 r_{\exp }$ & 0.998 & 0.997 & 0.997 & 1.000 & 1.000 \\
$1.10 r_{\exp }$ & 0.997 & 0.999 & 0.999 & 0.997 & $1.10 r_{\exp }$ \\
$\cos \theta(\mathrm{CH})$ & $0.90 r_{\exp }$ & $0.95 r_{\exp }$ & $1.00 r_{\exp }$ & $1.05 r_{\exp }$ & \\
\hline $0.90 r_{\exp }$ & 1.000 & & & & \\
$0.95 r_{\exp }$ & 0.836 & 1.000 & & & \\
$1.00 r_{\exp }$ & 0.842 & 0.964 & 1.000 & & \\
$1.05 r_{\exp }$ & 0.658 & 0.807 & 0.701 & 1.000 & \\
$1.10 r_{\exp }$ & 0.829 & 0.879 & 0.848 & 0.817 & \\
\hline \hline
\end{tabular}




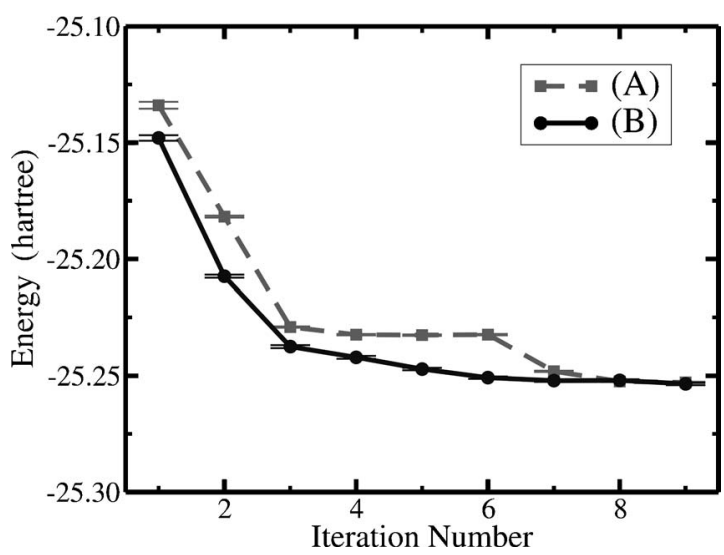

FIG. 2. The energy of $\mathrm{BH}$ at $r_{\exp }$ at various stages of parameter optimization. (A) Newton's method for 1-6 and Newton's method with SD for 6-9. (B) Initial SD for 1-3 and Newton's method for 3-9.

distance, the bond distance was changed, and the MO coefficients corresponding to this bond distance were introduced. Then, the Jastrow parameters were reoptimized at this new bond distance. This method makes it possible to reduce the CPU time for the calculation at other bond distances once the parameter set is optimized at one bond distance. This approach is effective because the Jastrow parameter sets at different bond distances can be quite similar, as measured by the cosine similarity ${ }^{19}$ between Jastrow parameter sets,

$$
\cos \theta=\frac{\mathbf{Q}_{m} \cdot \mathbf{Q}_{n}}{\sqrt{\mathbf{Q}_{m} \cdot \mathbf{Q}_{m}} \sqrt{\mathbf{Q}_{n} \cdot \mathbf{Q}_{n}}},
$$

which is close to unity if two vectors are similar. This is certainly the case for the Jastrow parameter sets of $\mathrm{HF}$ at various bond distances, as shown in Table III. This approach seems to be useful for the molecular-dynamics (MD) simulation coupled with QMC, proposed by Grossman and Mitas. ${ }^{20}$ On the other hand, in the case of $\mathrm{CH}, \mathrm{NH}$, or $\mathrm{OH}$, it was problematic to apply this method, and we had to optimize the parameters from the beginning for all bond distances. The cosine similarity values in the case of $\mathrm{CH}$ are shown in Table III, when the parameters are optimized separately from scratch for all bond distances. If the parameters of $\mathrm{HF}$ at each bond distance are optimized from scratch, the cosine similarity values are around 0.9 for the parameter sets optimized at different bond distances, and similar energies can be obtained with different sets of parameters.

The energy of $\mathrm{BH}$ at $r_{\exp }$ at various stages of parameter optimization is shown in Fig. 2. If the SD steps are used for initial stages of parameter optimization (B), Newton's method with SVD converges to the lowest energy after several iterations. If the initial SD steps are not used (A), New- ton's method is somewhat difficult to apply due to the large error bars of the Hessian components. In this case, it was necessary to set the SVD threshold somewhat high and to calculate for a long period of time. Within this approach, using only Newton's method with SVD does not yield fully optimized energy. The simultaneous application of Newton's method and SD (steps 6-9) was very useful in this case for more thorough minimization.

Forces were computed for each monohydride and carbon monoxide at each bond length in VMC and DMC. The force result for HF is shown in Fig. 1, where the slopes of the line segments superimposed on the energy result correspond to the negative of the calculated forces. The calculated forces of HF are shown in Table IV, together with the values obtained by fitting energy results to a parabola and then calculating the slopes. The force at $0.90 r_{\exp }$ is larger than the magnitude of the slope of the parabola obtained from the energy result, while the magnitude of the force at $1.10 r_{\exp }$ is smaller than the parabola tangent, which clearly shows the deviation of the calculated forces from harmonic behavior due to anharmonicity.

The approximate shape of the anharmonic potential can be described by the Morse potential, ${ }^{21}$

$$
V(r)=D_{e}\left[1-e^{-\beta\left(r-r_{e}\right)}\right]^{2},
$$

and this was used in the fitting of the QMC results to calculate the properties of diatomic molecules. The $v$ th energy level of the Morse potential with reduced mass $\mu$ is

$$
\frac{E}{h c}=\omega_{e}\left(v+\frac{1}{2}\right)-\omega_{e} x_{e}\left(v+\frac{1}{2}\right)^{2},
$$

where the harmonic vibrational frequency is given by $\omega_{e}$ $=\beta\left(100 D_{e} h / 2 \pi^{2} c \mu\right)^{1 / 2}$ and the anharmonicity constant by $\omega_{e} x_{e}=\left(100 h \beta^{2} / 8 \pi^{2} \mu c\right)$. In this equation, $\omega_{e}, D_{e}$, and $\beta$ have the unit of $\mathrm{cm}^{-1}$ and other constants are in Système International (SI) units.

Since we performed the QMC calculations at a small number of bond distances, it is advantageous to use the energy and force results simultaneously in the fitting to the Morse potential, which can be accomplished by minimizing the following merit function:

$$
\chi^{2}(\mathbf{a})=\sum_{i=1}^{N}\left[\frac{E_{i}-E\left(r_{i} ; \mathbf{a}\right)}{\sigma_{E, i}}\right]^{2}+\sum_{i=1}^{N}\left[\frac{F_{i}-F\left(r_{i} ; \mathbf{a}\right)}{\sigma_{F, i}}\right]^{2} .
$$

Here $\mathbf{a}$ is a parameter vector whose components are $D_{e}, \beta$, and $r_{e}$, and the following functional forms were used:

$$
E(r ; \mathbf{a})=D_{e}\left\{\left[1-e^{-\beta\left(r-r_{e}\right)}\right]^{2}-1\right\}+\left(E_{A}+E_{B}\right),
$$

TABLE IV. Forces obtained from the slope of parabolic potential-energy fits and from the direct calculation for HF.

\begin{tabular}{lllrrr}
\hline \hline Force & $0.90 r_{\exp }$ & $0.95 r_{\exp }$ & $1.00 r_{\exp }$ & $1.05 r_{\exp }$ & $1.10 r_{\exp }$ \\
\hline VMC (parabola) & $0.113(11)$ & $0.057(6)$ & $0.001(3)$ & $-0.055(6)$ & $-0.111(11)$ \\
VMC (direct) & $0.147(1)$ & $0.060(1)$ & $-0.002(1)$ & $-0.050(1)$ & $-0.076(2)$ \\
DMC (parabola) & $0.110(4)$ & $0.056(2)$ & $0.002(1)$ & $-0.051(2)$ & $-0.105(4)$ \\
DMC (direct) & $0.168(1)$ & $0.077(1)$ & $0.015(1)$ & $-0.033(1)$ & $-0.064(1)$ \\
\hline \hline
\end{tabular}




$$
F(r ; \mathbf{a})=-2 D_{e} \beta\left[1-e^{-\beta\left(r-r_{e}\right)}\right] e^{-\beta\left(r-r_{e}\right)} .
$$

The expression for the energy has been modified to produce correct dissociation energy $D_{e}$, and $E_{A}$ and $E_{B}$ are VMC or DMC energies of atoms $A$ and $B$. The equilibrium bond lengths $\left(r_{e}\right)$, harmonic vibrational frequencies $\left(\omega_{e}\right)$, and anharmonicity constants $\left(\omega_{e} x_{e}\right)$ for all first-row monohydride molecules and carbon monoxide calculated by fitting energy and force results are summarized in Table I, along with the experimental data. ${ }^{22}$ The experimental energies are corrected by adding zero point energies. Our calculations agree well with the experimental results.

Each energy or force data point has an error bar associated with it, so we followed a simple procedure to estimate how the calculated error bars translate into uncertainty in other quantities such as equilibrium bond length, harmonic vibrational frequency, and anharmonicity constant. A large set of synthetic data points was stochastically generated, such that the average value at each bond length agrees with that obtained from QMC with the standard deviation the same as the error bar given by the QMC calculation. By computing the averages and standard deviations of the equilibrium bond length $\left(r_{e}\right)$, harmonic vibrational frequency $\left(\omega_{e}\right)$, and anharmonicity constant $\left(\omega_{e} x_{e}\right)$ for the synthetic data sets, the error bars of $r_{e}, \omega_{e}$, and $\omega_{e} x_{e}$ could be estimated. The error bars of the last digit thus calculated are shown in parentheses.

\section{CONCLUSIONS}

The force calculation method combining energy minimization, Pulay's corrections, and a renormalized HellmannFeynman estimator worked well with all the first-row hydride molecules and carbon monoxide with small extra effort.

The energy minimization method in VMC is useful, but it requires an effective optimization scheme. The addition of steepest descents to the initial steps and to the subspace neglected by Newton's method with SVD seems to be advantageous for the molecular systems we investigated.

We could calculate accurate harmonic vibrational frequencies and anharmonicity constants of diatomic molecules by fitting QMC results to the Morse potential, achieving excellent agreement between QMC calculations and experiment for these vibrational parameters.

\section{ACKNOWLEDGMENTS}

This work was supported by the Air Force Office of Scientific Research, Air Force Material Command, USAF, under Grant No. FA9550-04-1-0077. Computational support was provided by the Defense University Research Instrumentation Program, and by the NSF CRIF program, Grant CHE-0131132.

${ }^{1}$ R. Assaraf and M. Caffarel, J. Chem. Phys. 113, 4028 (2000).

${ }^{2}$ R. Assaraf and M. Caffarel, J. Chem. Phys. 119, 10536 (2003).

${ }^{3}$ C. Filippi and C. J. Umrigar, Phys. Rev. B 61, R16291 (2000).

${ }^{4}$ M. Casalegno, M. Mella, and A. M. Rappe, J. Chem. Phys. 118, 7193 (2003).

${ }^{5}$ S. Chiesa, D. M. Ceperley, and S. Zhang, Phys. Rev. Lett. 94, 036404 (2005).

${ }^{6}$ X. Lin, H. Zhang, and A. M. Rappe, J. Chem. Phys. 112, 2650 (2000).

${ }^{7}$ W. H. Press, S. A. Teukolsky, W. T. Vetterling, and B. P. Flannery, Numerical Recipes in Fortran 77 (Cambridge University Press, Cambridge, 1992).

${ }^{8}$ P. E. Cade and W. M. Huo, J. Chem. Phys. 47, 614 (1967).

${ }^{9}$ P. E. Cade and W. M. Huo, At. Data Nucl. Data Tables 15, 1 (1975).

${ }^{10}$ M. J. Frisch, G. W. Trucks, H. B. Schlegel et al., GAUSSIAN 98, Revision A.11.2, Pittsburgh, PA, 2001.

${ }^{11}$ A. Lüchow and J. B. Anderson, J. Chem. Phys. 105, 7573 (1996).

${ }^{12}$ G. Morosi, M. Mella, and D. Bressanini, J. Chem. Phys. 111, 6755 (1999).

${ }^{13}$ S. F. Boys and N. C. Handy, Proc. R. Soc. London, Ser. A 310, 43 (1969).

${ }^{14}$ K. E. Schmidt and J. W. Moskowitz, J. Chem. Phys. 93, 4172 (1990).

${ }^{15}$ B. L. Hammond, W. A. Lester, Jr., and P. J. Reynolds, Monte Carlo Methods in Ab Initio Quantum Chemistry (World Scientific, Singapore, 1994).

${ }^{16}$ P. J. Reynolds, D. M. Ceperley, B. J. Alder, and W. A. Lester, Jr., J. Chem. Phys. 77, 5593 (1982).

${ }^{17}$ A. Lüchow and J. B. Anderson, J. Chem. Phys. 105, 4636 (1996).

${ }^{18}$ P. Pulay, Mol. Phys. 17, 197 (1969).

${ }^{19}$ G. Salton and M. McGill, Introduction to Modern Information Retrieval (McGraw-Hill, New York, 1983).

${ }^{20}$ J. C. Grossman and L. Mitas, Phys. Rev. Lett. 94, 056403 (2005).

${ }^{21}$ P. M. Morse, Phys. Rev. 34, 57 (1929).

${ }^{22}$ K. P. Huber and G. Herzberg, Constants of Diatomic Molecules, Molecular Spectra and Molecular Structure Vol. IV (Van Nostrand Reinhold Company, New York, 1979). 